\title{
Renaissance Editions of Classical Texts in the Library of A. J. Bell
}

\author{
LARRY PFAFF
}

A catalogue of the Erasmus collection in the Centre for Reformation and Renaissance Studies of Victoria University, Toronto, appeared in 1971 (Renaissance and Reformation, VII, No. 2). Professor Douglas Bush's portrait of the collector A.J. Bell was reprinted in the same issue.

Bell's collection was not confined to Erasmus. ${ }^{1}$ It included as well early editions of Trissino's Sophonisba (Vicenza, 1529), Paolo Giovio's Elogia virorum bellica virtute illustrium and Elogia virorum literis illustrium (Basel, 1575-7), and a copy of Juvencus' Evangeliorum Libri IV (Leipzig, 1511) with annotations which have been identified as those of Philip Melanchthon.

Above all Bell was a Classical scholar but his remarkable collection of early editions of Greek and Latin Classical texts has been overlooked largely because it is dispersed in several sections of the Victoria College Library: the majority are located in the Centre for Reformation and Renaissance Studies, others are among the unprocessed material of the Victoria College Library, and a few others have not been located. Since none have been catalogued according to the Library of Congress system they are not to be found in either the Victoria University or the Robarts Library union catalogues.

The following preliminary short-title listing of editions of the Classics published from approximately 1490 to 1650 attempts to reassemble this important part of A.J. Bell's collection. The works of certain Christian Fathers, such as St. Jerome, who are important to Classical students have also been included.

Bell also owned many editions from the late seventeenth and eighteenth centuries which included Renaissance commentaries, for example,

1 For a general survey of Bell's collection see my article, "Andrew James Bell: a great Toronto book-collector," to appear in the forthcoming Volume XV, 1976 of the Papers of the Bibliographic Society of Canada. I wish to thank once again Miss Sybille Pantazzi and the staff of the E.J. Pratt Library and the Centre for Reformation and Renaissance Studies for their assistance. 
the Havercamp variorum edition of Lucretius' De Rerum Natura (Leyden, 1725), but to include all of these would unduly prolong this list.

The list which follows is based on Bell's own catalogue and wherever possible his entry and the title-page of the book were checked with the entry in the printed catalogues of the British Museum and the Bibliothèque Nationale. Twenty books have not been located: some of these may be bound with other early books; some may have been inadvertently sold in a series of sales held at Victoria College in 1968-9. The compiler would be grateful for any information regarding the present location of these books. For each entry in the list the location is indicated:

$$
\begin{array}{ll}
\text { CRRS } & - \text { Centre for Reformation and Renaissance Studies, } \\
& \text { Victoria University } \\
\text { EJPL } & - \text { E.J. Pratt Library, Victoria College, Toronto } \\
\dagger & - \text { location unknown }
\end{array}
$$

\section{AESCHYLUS}

... Prometheus, cum interpretatione Mathiae Garbitii Illyrici. Basileae, Per I. Oporinum, $1559.8^{\circ}$. CRRS

\section{ANTIQUI RHETORES LATINI}

Rutillius Lupus, Aquila Romanus, Iulius Rufinianus, etc. Parisiis, Plantaniana, I599. $4^{\circ}$. CRRS

APULEIUS, Lucius Madaurensis

Opera omnia quae exstant Geuerhartus Elmenhorstius... recensuit, librumque emendationum ... adiecit. Francofurti, In officina Wecheliana apud D. et D. Aubrios et C. Schleichium, 1621. $8^{\circ}$. EJPL

ARISTIDES, Aelius

... Orationes. Florentiae, Sumptibus P. Juntae, 1517. fol. $\dagger$

ARISTOTLE

... Opera ... omnia. Per Des. Eras. Roterodamum. Basileae, Per Io. Bebelium \& Mich. Isingritium, 1550. fol. 2 vols. $\dagger$

ARRIANUS, Flavius

... De ascensu Alexandri. Venetiis, In aedibus B. Zanetti, 1535. $8^{\circ}$. CRRS

... De rebus gestis Alexandri magni regis Macedonium libri octo Bartholomaeo Facio ... interprete. Lugduni, Apud S. Gryphium, 1552. $12^{\circ}$. CRRS

AUGUSTINUS, Aurelius

De natura et gratia liber unus. Item de spiritu \& litera liber unus. Norembergae, 1524. $8^{\circ}$. CRRS

Opuscula plurima ... Argentinae, Impensis et opera M. Flach, 1491. fol. CRRS

AURELIUS ANTONINUS, Marcus

... De se ipso et se ipsum libri XIl. Xylandri versionem locis plurimis emendavit, \& novam fecit; in Antonini libros notas \& emendationes adiecit Mericus Casaubonus. Londini, Typis M. Flesher, Sumptibus R. Mynne, 1643. $8^{\circ}$. EJPL 
AUSONIUS, Decimus Magnus

Omnia opera, nuper maxima diligentia recognita atque excusa. Florentiae, Sumptu P. Juntae, $1517.8^{\circ}$. CRRS

BOETHIUS, Anicius Manlius Severinus

De consolatione philosophiae. Coloniae, Per Johannem Koelhoff, 1488. fol. CRRS

De consolatione philosophiae et de disciplina scholarium. Venetiis, Per Johannem et Gregorium de Gregoriis, 1497. fol. CRRS

Opera. Venetiis, Per Johannem et Gregorium de Gregoriis, 1499. fol. CRRS

CASSIODORUS, Flavius Magnus Aurelius

Variarum libri XII \& Chronicon, ad Theodericum regem. Iordani Episcope Ravennatis De origine actibusque Getarum liber I ... Enodii Ticinensis Episcopi Panegyricus Theoderico dictus. G. Fornerii ... notae in libros Variarum. Parisiis, Apud S. Nivellium, $1583.4^{\circ} . \dagger$

Variarum libri XII \& Chronicon ... G. Fornerii ... notae in lib. Variar. His de novo accesserunt Francisci Modii Argumenta ... Petri Brossei in Cassiodorum Animadversiones. Gnomologia Cassiodorana ... Lugduni, Apud I. Chouët, 1595. $8^{\circ}$. CRRS

"CATO, Dionysius"

Praecepta moralia recognita atque interpretata ab Erasmo Roterodamo. Argentorati, Ex aedibus Schurerianis, $1516.4^{\circ}$. CRRS

Disticha de moribus ad filium cum D. Erasmi Roterodami brevi expositione; eadem graece reddita per Jos.Scaligerum, etc. Lugduni Batavorum, Apud Andream Cloucquium, 1626. $8^{\circ}$. CRRS

Disticha de moribus ad filium; cum D. Erasmi Roterodami brevi expositione; eadem graece reddita per Jos. Scaligerum ... accedit Marci Zuerii Boxhornii De distichis Catonis dissertatio. Amstelodami, Apud Joannem Janssonium, 1646. fol. CRRS

Disticha de moribus, cum scholiis Des. Erasmi Roterodami. Londini, Typis A.H. impensis Societatis Stationariorum, 1661. $8^{\circ}$. CRRS

CATO, Marcus Porcius

Libri de re rustica. M. Catonis lib. I. M. Terentii Varronis lib. III. L. Iunii Moderati Columellae lib. XII ... Palladii lib. XIIII, etc. Venetiis, In aedibus Aldi \& Andreae Soceri, $1514.4^{\circ} . \dagger$

CATULLUS, Gaius Valerius

Catulli, Tibulli, Propertii, nova editio. Iosephus Scaliger recensuit. Eiusdem in eosdem Castigationum liber ... Antverpiae, Apud A. Radaeum, 1582. $8^{\circ}$. CRRS

C. Val. Catulli, Albii Tibulli, Sex. Aur. Propertii, opera omnia quae extant. Cum variorum doctorum virorum commentariis, notis... Lutetiae, Ex officina C. Morelli, 1604. fol. CRRS

Catullus, Tibullus, et Propertius, et quae sub Galli nomine circumferunter; cum selectis variorum commentariis accurante Simone Abbes Gabbema. Trajecti ad Rhenum, Typis G. à Zijll, \& T. ab Ackersdijck, 1659. $8^{\circ}$. EJPL

Catullus, Tibullus, et Propertius, ex recensione Joannis Georgii Graevii, cum notis integris Jos. Scaligeri, M. Ant. Mureti, Achill. Statii, Roberti Titii, Hieronymi 
Avantii, Jani Dousae partris, \& filii, Theodori Marcilii, nec non selectis aliorum. Trajecti ad Rhenum, Ex officina R. à Zyll, 1680. $8^{\circ}$. EJPL

Caius Valerius Catullus et eum Isaaci Vossii observationes. Prostant apud Isaacum Littleburii Bibliopolam Londinensem, 1684. $4^{\circ}$. EJPL

CICERO, Marcus Tullius

Tusculanae quaestiones. Lugduni, S. Gryphium, 1541. $8^{\circ}$. CRRS

Officia diligenter restituta. Eiusdem de Amicitia, \& Senectute Dialogi singuli. Cum ann. Erasmi Roterodami, \& Ph. Melanchthonis; Item, Annotation. Bartholomaei Latomi in Paradoxa. Parisiis, Apud S. Colinaeum, 1543. 16 . CRRS

De officiis libri III. Commentariis Erasmi... Melanchthonis ... Amberbachii, \& Francisci Maturantii ... eiusdem, De Senectute, De Amicitia, Paradoxa, Somnium Scipionis, cum adnotationibus ... D. Erasmi ... Lugduni, Apud T. Paganum, 1556. $4^{\circ}$. CRRS

Contra Catilinam orationes quatuor. Portii Latronis declamatio contra L. Sergium Catilinam. etc. Coloniae, Martinus Gymnicus excudebat, 1563. $8^{\circ}$. CRRS

Pro T. Annio Milone Oratio, Philippi Melancthonis, \& Bartholomaei Latomi adnotationibus ... Coloniae, Martinus Gymnicus excudebat, 1563. $8^{\circ}$. CRRS

Orationes II. Altera pro A. Licinio Archia poëta, altera pro M. Marcello. Cum artificio, dispositione, ac paraphrasi Philippi Melancthonis. Coloniae, Martinus Gymnicus excudebat, $1563.8^{\circ}$. CRRS

Epistolae ad familiares libri XVI. Pauli Manutii scholia, quibus et loci familiarum epistolarum difficiliores explicantur... In Libera Argentina, Excudebat Iosias Rihelius, $1569.8^{\circ}$. CRRS

Epistolae ad familiares. Philippi Melanchthonis argumenta \& scholia. Ioach. Camerarii \& Eras. Roterod. locorum obscuriorum recognitio verba Graeca Latinis expressa ... Francofurti, Apud Martinum Lechlerum impensis haeredum Christiani Egenolphi, $1592.8^{\circ}$. CRRS

CLAUDIANUS, Claudius

Quae exstant: ex emendatione Nicolai Hensy. Amstelodami, Typis S. Elzevirii, 1650. $12^{\circ}$. EJPL

\section{CURTIUS RUFUS, Quintus}

Historia Alexandri Magni, cum notis selectiss. variorum, Raderi, Freinshemii, Loccenii, Blancardi, etc. Lugduni Batavorum, Apud Iohannem Elsevirium, 1658. $8^{\circ}$ CRRS

\section{DEMOSTHENES}

... Gnomologiae, id est sententiae collectaneae, \& Similia ex Demosthenis orationibus $\&$ epistolis in certa virtutum ac vitiorum capita collectae. Authore Ioanne Loino Illesiensi. Parisiis, Ex officina Viduae Mauricii, 1551. $8^{\circ}$. CRRS

Demosthenis et Aeschinis principium Graeciae oratorum opera, cum utriusq; autoris vita, \& Ulpani commentariis, novisq; scholiis, ex quarta, eaque postrema recognitione, graeco-latine: sex codicum impressorum ... duorum manuscriptorum ... collatione, à mendis repurgata, variis lectionib. adaucta, annotationib. illustrata: per Hieronymum Wolfium ... Francofurti, Apud Claudium Marnium, \& haeredes Iohannes Aubrii, 1604. fol. EJPL 


\section{DICTYS CRETENSIS}

Dictys Cretensis, et Daretis Phrygii, De bello Troiano historia. Declamationes tres Libanii Sophistae, eiusdem ferè argumenti. Lugduni, Apud Antonium Gryphium, 1569. $16^{\circ} . \dagger$

\section{DIODORUS SICULUS}

... Bibliothecae historicae libri quindecim de quadraginta. Genevae, Henricus Stephanus, 1559. fol. CRRS

\section{DION CHRYSOSTOM}

... Orationes LXXX ... Venetiis, Apud Federicum Turrisanum, 1551. $8^{\circ}$. CRRS

DIONYSIUS of Halicarnassus

Antiquitatum sive originum Romanorum libri X. Sigismundo Gelenio interprete ... Basileae, Froben, 1549. fol. CRRS

\section{EROTIANUS}

... Vocum quae apud Hippocratem sunt collectis, cum annotationibus Bartholomaei Eustachii ... eiusdem Eustachii libellus de multitudine. Venetiis, Apud L.A. Juntam, $1566.4^{\circ}$. $\dagger$

\section{EUCLID}

Elementorum sex libri priores magnam partem novis demonstrationibus adornati, opera et studio Henrici Coetsii. Lugduni Batavorum, D. a Gaesbeek, 1692. $8^{\circ}$. EJPL

\section{EUNAPIUS}

De vitis philosophorum et sophistarum liber. Coloniae Allobrogum, 1616. $8^{\circ} . \dagger$

\section{EURIPIDES}

... Tragoediae duae, Hecuba \& Iphigenia in Aulide, Latinae factae, Des. Erasmo Roterodamo interprete. Basileae, Apud Ioannem Frobenium, 1524. $8^{\circ}$. CRRS

FLORUS, Lucius Annaeus

Rerum Romanarum libri IV. Cl. Salmasius addidit L. Ampelium e cod. MS. nunquam antehac editum. Lugduni Batavorum, Apud J. Elzevirium, 1657. 12 ${ }^{\circ}$. CRRS

\section{GALEN}

Omnia, quae extant, in Latinum sermonem conversa ... Basileae, Per H. Frobenium et N. Episcopium, 1561. fol. [part 3 of 11] EJPL

GELLIUS, Aulus

Noctes Atticae. Lugduni, Apud A. Gryphium, 1566. $8^{\circ}$. CR RS

\section{HELIODORUS}

Aethiopicorum libri X. Collatione MSS. Bibliothecae Palatinae \& aliorum emendati, $\&$... aucti ... cura et labore Danielis Parei. Francofurti, Impensis G. Fitzeri, $1631.8^{\circ}+$

\section{HERMOGENES}

... Ars oratoria absolutissima et libri omnes. Cum nova versione Latina ... et commentariis G. Laurentii. Coloniae Allobrogum, Apud P. Aubertum, 1614. $8^{\circ}$. EJPL 


\section{HERODIAN}

... Histor. lib. VIII cum Angeli Politiani interpretatione ... Eiusdem H. Stephani emendationes quorundam Graeci contextus locorum ... Genevae, H. Stephanus, $1581.4^{\circ}$. CRRS

\section{HERODOTUS}

... Historiae libri IX, interprete Laurentio Valla. Eiusdem Herodoti libellus de vita Homeri, interprete Conrado Heresbachio. Basileae, per Sebastianum Henricpetri, $1583.8^{\circ}$. CRRS

\section{HESIOD}

... Opera et dies, cum interpretatione Latina et scholiis ... autore Mathia Garbitio ... Basileae, Per J. Oporinum, 1559. $8^{\circ}$. CRRS

HIERONYMUS, Divus, Stridonensis

Opera omnia, cum argumentis et scholiis D. Erasmi. Basileae, In aedibus Io. Frobenii, 1516. Fol. 9 vols. in 4. Vols. 3, 4 lacking. CRRS

Opus epistolarum divi Eusebii Hieronymi Stridonensis, una cum scholiis Des. Erasmi Rotero. Lugduni, In officina Ioannis Crespini, 1528. $4^{\circ}$. CRRS

Omnes quae extant D. Hieronymi Stridonensis lucubrationes, additis una pseudepigraphis et alienis, scriptis ipsius admixtis, in novem tomos, per Des. Erasmum Roterodamum digestae ... Basileae, Froben, 1553. fol. 10 vols. CRRS

\section{HISTORIAE AUGUSTAE SCRIPTORES}

Ex recognitione D. Erasmi, C. Suetonius Tranq., Dio Cassius, Aelius Spartianus, etc. Basileae, Apud Ioannem Frobenium, 1518. fol. CRRS

C. Suetonius Tranquillus... Annotationes D. Erasmi Rot. \& Baptistae Egnatii in vitas Caes. Basileae, Froben, 1546. fol. CRRS

HORATIUS FLACCUS, Quintus

Omnia poemata cum ... interpretibus Acrone, Porphyrione, Jano Parrhasio, Antonio Mancinello, necnon Jodoco Badio Ascensio. Venetiis, Apud J.M. Bonellum, 1562. fol. CRRS

Ex fide ... decem librorum manuscriptorum opera Dionysii Lambini ... emendatus: ab eodemque commentariis ... illustratus. His adiecimus J.M. Bruti in quatuor libros ... Venetiis, Apud P. Manutium, 1566. $4^{\circ} . \dagger$

Opera, grammaticorum XL ... partim iustis commentariis, partim succinctis annotationibus ... in unum corpus collectis, illustrata. Basileae, S. Henricpetri, 1580. fol. EJPL

Cum commentariis ... commentatoris veteris, et J. Cruquii, etc. Lugduni Batavorum, Ex officina Plantiniana Raphelengii, 1611. $4^{\circ}$. CRRS

\section{IAMBLICHUS}

Iamblichus ... in Nicomachi Geraseni arithmeticam introductionem, et de fato ... in Latinum sermonem conversus ... a S. Tennulio. Accedit J. Camerarii explicatio in duos libros Nicomachi etc. Daventriae, Arnhemiae, 1668,7. $4^{\circ} . \dagger$ [Inscribed and annotated by Richard Bentley] 
unus ... castigatus ab Erasmo Roterodamo. Coloniae Agrippina, In aedibus Eucharii Cervicorni, 1524. fol. CRRS

JUVENALIS, Decimus Junius

D.J. Juvenalis et A. Persij Flacci Satyrae; cum veteris scholiastae et variorum commentariis. Accurante C. Schrevelio. Lugduni Batavorum, 1671. $8^{\circ}$. EJPL

\section{LIBANIUS SOPHISTA}

Declamatiunculae aliquot eaedemque Latinae per Des. Erasmum Rot. cum duabus orationibus Lysiae itidem versis incerto interprete \& aliis nonnullis. Basileae, Ex aedibus Ioannis Frobenii, 1522. $4^{\circ}$. CRRS

Declamatio Libanii sophistae, latina facta Erasmo Roterodamo interprete. Coloniae, Ioanne Sotere, $1525.8^{\circ}$. CRRS

LUCANUS, Marcus Annaeus

Pharsalia: cum ... annotatione Petri de Ponte... Parrhisiis, G. le Rouge, Expensis vero Dionisii Roce, $1512.8^{\circ} . \dagger$

Pharsalia ... Additae sunt ... H. Grotii notae, ... auctae, ... et T. Farnabii. Amsterodami, Apud J. Blaeuw, 1643. $12^{\circ}$. EJPL

De bello civile, cum H. Grotii, Farnabii, notis integris et variorum selectiss. Accurante C. Schrevelio. Lugduni Batavorum, Apud F. Hackium, 1669. $8^{\circ} . \dagger$

\section{LUCIAN SAMOSATENSIS}

Saturnalia, Cronosolon ... Epistolae saturnalis, De luctu, Abdicatus, etc. Des. Erasmo Roterodamo interprete, ex posteriori recognitione. Basileae, Frobenius, 1517. $4^{\circ}$. CRRS

Saturnalia ... Des. Erasmo Roterodamo interprete. Basileae, Apud Joannem Frobenium, 1521. fol. CRRS

.. Omnia quae extant, cum Latina interpretatione. J. Bourdelotius ... emendavit ... Adjectae sunt eiusdem Bourdelotii, T. Marcilii, G. Cognati notae ... Lutetiae Parisiorum, J. Bertault, 1615. fol. CRRS

Philosophi opera. Lutetiae Parisiorum, Apud P. Ludovicum, 1615. fol. CRRS

LUCRETIUS CARUS, Titus

De rerum natura. Venetiis, Per T. de Ragazonibus de Asula, 1495. $4^{\circ}$. EJPL

De rerum natura libri sex. A.D. Lambino, ... ex auctoritate quinque codicum manu scriptorum, emendati, ... et praeterea ... commentariis illustrati. Parisiis, In G. Rovillii et P.G. Rovillii .... aedibus, 1563. $4^{\circ}$. CRRS

De rerum natura libri sex, ad postremam Oberti Gifanii ... emendationem restituti ... Lugduni Batavorum, Ex officina Plantiniana, Apud F. Raphelengium, 1595. $8^{\circ}$ CRRS

De rerum natura libri sex, notis ... ex ipso auctore ... illustrati, cum lexico ... omnium elegantiarum Lucretianarum, cura ... D. Parei ... Francofurti, Impensis G. Fitzeri excudebat W. Hofmannus, 1631. $8^{\circ}$. EJPL

\section{LYSIAS}

Orationes XXXIV, quae de CCC reliquae sunt, nunc primum de graecis latine redditae et politicis notis illustratae a Jodoco Vander-Heidio ... Hanoviae, Typis Wechelianis, $1615.8^{\circ}$. EJPL 
MACROBIUS, Ambrosius Theodosius

In somnium Scipionis, lib. II. Saturnaliorum, lib. VII. Ex variis, ac vetustissimis codicibus recogniti, \& aucti. Lugduni, Apud haered. S. Gryphii, 1560. $16^{\circ}$. CRRS

Opera. Accedunt notae integrae Isaacii Pontani, Joh. Meursii, Jacobi Gronovii. Lugduni Batavorum, Ex officina A. Droude, 1670. $8^{\circ} . \dagger$

MANILIUS, Marcus

Astronomicon libri quinque, J. Scaliger recensuit ac pristino ordini suo restituit. Eiusdem J. Scaligeri commentarius in eosdem libros et castigationum explicationes. Lutetiae, R. Stephanus, 1579. $8^{\circ}$. CRRS

MARTIALIS, Marcus Valerius

Epigrammatum libri XV. Cum variorum doctorum virorum commentariis ... Cum indice omnium verborum J. Langii ... Lutetiae Parisiorum, Apud M. Sonnium, 1617. fol. CRRS

MELA, Pomponius

Pomponius Mela. Julius Solinus. Itinerarium Antonini Aug. Vibius Sequester. P. Victor de regionibus urbis Romae. Dionysius Afer de situ orbis, Prisciano interprete. Florentiae, Per Haeredes P. Juntae, 1519. $8^{\circ}$. CRRS

OVIDIUS NASO, Publius

Vita per Aldum, ex ipsius libris excerpta, Heroidum epistolae, Amorum libri III, De arte amandi libri III, De remedio amoris libri II, ... Venetiis, In aedibus Aldi et Andreae soceri, 1515. $8^{\circ}$. [Vol. 2 of the Aldine edition of the Opera] EJPL

Metamorphosis cum luculentissimis Raphaelis Regii enarrationibus... Venetiis, Leonardo Lauredano, 1517.4 . CRRS

Commentarius Erasmi Roterodami in nucem Ovidii. Lutetiae, Apud Carolum Stephanum, $1555.8^{\circ}$. CRRS

Heroidum epistolae. Amorum libri III. De arte amandi libri III. De remedio amoris libri II. Aliaque huius generis ... omnia ex Andreae Naugerii castigatione. Guidonis Morilloni argumenta in epistolas. Lugduni, Apud A. Gryphium, 1588. $16^{\circ}+$

\section{PERSIUS FLACCUS, Aulus}

In A. Flacci Persii satyras sex, quatuor ... commentarii. Valentini, Volsci, Engentini, Foquelini. Labore et studio J.T. Freigii nunc primum coniuncte editi. Basileae, Ex officina Petri Persiae, 1578. $4^{\circ}$. CRRS

Satirarum liber. I. Casaubon recensuit, \& commentario libro illustravit. Tertia editio, auctior \& emendatior ex ipsius authoris codice: cura et opera Merici Casauboni. Londini, Typis M. Flesher: sumptibus R. Mynne, 1647. $8^{\circ}$. CRRS

PETRONIUS ARBITER, Titus

Satyricon. Lugduni Batavorum, Ex officina Plantiniana Raphelengii, 1614. $32^{\circ}$. CRRS

Satyricon cum uberioribus commentarii instar notis... Amsterodami, Apud G.I. Caesium, 1626. $12^{\circ}$. CRRS

PHALARIS

Phalaridis \& Bruti epistolae ... Graecè \& Latinè. Aphthonii Sophistae Progymnas- 
mata. Francisco Scobario interprete, cum notis ex commentariis Hadamarii ... Theognidis Phocylidis, Pythagorae, Solonis ... opera F. Sylburgii. Heidelbergae, Apud Hieronymum Commelinum, 1597. $8^{\circ}$. CRRS

PINDAR

Olympionikai, Pythionikai, Nemeonikai, Isthmionikai, illustrati versione nova fideli ... Opera E. Schmidii. Witebergae, Sumptibus Z. Schurerii, 1616. $4^{\circ}$. CRRS

PLATO

Omnia opera. Cum commentariis Procli in Timaeum et Politica ... Basileae, Apud J. Valderum, 1534. fol. $\dagger$

Omnia divini Platonis opera, tralatione Marsilii Ficini, emendatione et ad graecum codicem collatione Simonis Grynaei ... Lugduni, Apud A. Vincentium, 1548. fol. CRRS

PLAUTUS, Titus Maccius

Elegantiarum ex Plauto et Terentio libri duo, Publii Syri mimographi \& poëtarum veterum sententiae selectae, cum Des. Erasmi Rot. et Georg. Fabr. Chem. expositionibus. Lipsiae, Imprimebat Ioannes Steinman, 1581.8 ${ }^{\circ}$. CRRS

Comoediae XX. superstites. J.P. Pareus ... restituit et notis perpetuis illustravit ... Francofurti, Impensis Jenae Rhodii, 1610. $8^{\circ}$. CRRS

Comoediae interpretatione et notis illustravit J. Operarius ... in usum Delphini. Parisiis, F. Leonard, 1679. $4^{\circ}$. EJPL

PLINIUS SECUNDUS, Caius

Historia Mundi ... Basileae, Froben, 1525. fol. CRRS

PLINIUS CAECILIUS SECUNDUS, Caius

Epistolarum libri X ... cum commentariis Joannis Mariae Catanaei ... Genevae, Apud Petrum \& Jacobum Chouët, 1625. $4^{\circ}$. CRRS

PLUTARCH

Libellus ... saluberrimis praeceptis refertus; Erasmo interprete. Basileae, Froben, $1516.4^{\circ}$. CRRS

Opuscula varia [Erasmo, etc. interpr.]. Genevae, Excudebat Henr. Stephanus, 1572. 3 vols. CRRS

POLYBIUS

... Historiarum libri qui supersunt. I. Casaubonus ... emendavit, Latine vertit, et commentariis illustravit. Francofurti, Apud C. Marnium, 1609. fol. EJPL

PRUDENTIUS CLEMENS, Aurelius

Opera ... notisque et indice accurato illustrata a Iohanne Weitzio ... Hanoviae, Typis Wechelianis apud haeredes Iohannis Aubrii, 1613. $8^{\circ}$. CRRS

Opera interpretatione et notis illustravit S. Chamillard. Parisiis, Apud Viduam Claudii Thiboust, 1687. $4^{\circ}$. EJPL

QUINTILIANUS, Marcus Fabius

Institutionum oratoriarum libri XII ... Eiusdem Declamationum liber. Basileae, Ex aedibus loannis Bebelii, 1529. fol. CRRS 
Institutionum oratoriarum libri XII. Declamationum eiusdem liber. Lugduni, Apud Seb. Gryphium, 1540. EJPL

SENECA, Lucius Annaeus

Tragoediae, pristinae integritati restitutae per exactissimi indicii viros, post avantium et philologum: D. Erasmum Roterodamum, Gerardum Vercellanum, Aegidium Maserium ... Explanatae ... tribus commentariis: G. Bernardino Marmita, ... Daniele Gaietano, ... lodoco Badio Ascensio. Parisiis, Impensis \& industria Ascensiana, 1514. CRRS

Ludus de morte Claudii Caesaris ... cum scholiis Beati Rhenani. Basileae, Apud Io. Frobenium, 1521. $8^{\circ}$. CRRS

Ludus de morte Claudii Caesaris ... cum scholiis Beati Rhenani. Basileae, Apud lo. Frobenium, 1522. $8^{\circ}$. CRRS

Opera quae exstant integris J. Lipsii, J. Fr. Gronovii et selectis variorum commentariis illustrata. Accedunt L. Fromondi in Quaestionum Naturalium libros Apokolokuntosin notae et emendationes ... Amstelodami, Apud D. Elsevirium, $1672.8^{\circ} .3$ vols. CRRS

SENECA, Marcus Annaeus \& Lucius Annaeus

Opera omnia, ab Andrea Schotto ad veterum exemplarium fidem castigata ... Genevae, P. Chouet, 1636. $8^{\circ}$. EJPL

SEVERUS, Sulpicius

Opera omnia cum ... commentariis. Accurante G. Hornio. Lugduni Batavorum, Apud F. Hackium, 1647. $8^{\circ}$. EJPL

SILIUS ITALICUS, Caius

Opus de bello punico secundo ... cura A. Nicandri castigatum ... Florentiae, Iuntae, $1515.8^{\circ}$. CRRS

SOLINUS, Caius Julius

Polyhistor, seu rerum orbis memorabilium collectanea. Coloniae, Apud E. Cervicornum et $\mathrm{H}$. Fuchs, $1520.4^{\circ}$. CRRS

Commentaria in C. Iulii Solini Polyhistoria, et Lucii Flori De Romanorum rebus gestis, libros, ac tabulam Cebetis ... Ioanne Camerte autore praeterea Pomponii Melae De orbis situ libri tres cum commentariis Ioachimi Vadiani ... Basileae, Per Henrichum Petri, 1557. fol. CRRS

STATIUS, Publius Papinus

Sylvarum libri quinque. Thebaidos libri duodecim. Achilleidos duo. Venetiis, In aedibus Aldi, 1502. $8^{\circ}$. CRRS

Quae exstant. C. Barthius recensuit ... Cygneae, Ex officina M. Göpneri, 1664-5. $4^{\circ} .2$ vols. $\dagger$

Sylvarum lib. V. Thebaidos lib. XII. Achilleidos lib. II. notis selectissimis ... illustrati a Johanne Veenhusen. Lugduni Batavorum, Ex officina Hackiana, 1671. $8^{\circ}$. EJPL

SUETONIUS TRANQUILIUS, Caius

[De vita Caesarum] cum Philippi Beroaldi et Marci Antonii Sabellici commentariis. Venetiis, Per Simonem cognomento Bevilaqua Papiensem, 1496. fol. CRRS 
Liber illustrium virorum. Argentorati, Ex aedibus Matthiae Schurerii, 1517. $4^{\circ}$. CRRS

Duodecim Caesares, ex Erasmi recognitione. Parisiis, Apud Simonem Colinaeum, 1535. $8^{\circ}$. CRRS

Duodecim Caesares, ex Erasmi recognitione. Parisiis, Apud Simonem Colinaeum, $1543.8^{\circ}$. CRRS

XII Caesares, T. Pulmanni ... opera et studio emendati. In eosdem annotationes ... ex vetustis vulgatisque libris, ab eodem collectae. Eiusdem C. Suetonii Tranquilli de illustribus grammaticis \& claris rhetoribus lib. II, cum Achillis Statii ... commentatione. I.B. Egnatii, D. Erasmi ... \& H. Loriti ... in Suetonum annotationes. Antverpiae, Ex officina C. Plantini, $1574.8^{\circ}$. CRRS

SYMMACHUS, Quintus Aurelius

Epistolarum ad diversos libri decem ... restituti cura et studio F. Jureti, cuius etiam notae adiectae sunt ... Parisiis, Apud N. Chesneau, 1580. $4^{\circ} . \dagger$

TERENTIUS AFER, Publius

Comoedias, una cum scholiis ex Donati, Asperi, et Cornuti commentariis decerptis ... indicata sunt diligentius carminum genera ... studio et opera Des. Erasmi ... Basileae, In officina Frobeniana, 1532. fol. CRRS

Comoedias una cum scholiis ex Donati ... Indicata sunt diligentius carminum genera \& in his incidentes difficultates, correcta quaedam \& consulum nomina, idque studio \& opera Des. Erasmi... Basileae, In officina Frobeniana, per Hier. Frobenium et Nic. Episcopium, 1538. fol. CRRS

TACITUS, Publius Cornelius

Annotationes Beati Rhenani, Alciati, Beroaldi, ac Aemylii Ferretti. 2 pt. Lugduni, Apud S. Gryphium, 1542,41. $8^{\circ}$. CRRS

VARRO, Marcus Terentius

In hoc volumine habentur haec: Nicolai Perotti Cornucopiae sive linguae latinae commentarii. M.T. Varronis De lingua latina libri tres, quartus, quintus, sextus. Eiusdem De analogia libri tres. S. Pompeii Festi undeviginti librorum fragmenta ... Venetiis, In aedibus Aldi et Andreae soceri, 1513. fol. $\dagger$

Opera quae supersunt. In lib. de ling. Lat. conjectanea J. Scaligeri recognita \& appendice aucta. In libros de re rust. notae eiusdem Jos. Scal. non antea editae. His adjuncti fuerunt A. Turnebi comment. in lib. de lingua Latina cum emendationibus A. Augustini. Item P. Victorii castigationes in lib. de re rustica. Genevae, H. Stephanus, $1573.8^{\circ} .2$ copies CRRS

Opera omnia quae extant. Cum notis J. Scaligeri, A. Turnebi, P. Victorii et A. Augustini. His accedunt tabula naufragii seu fragmenta eiusdem auctiora ... Durdrechti, J. Berewout, 1619. $8^{\circ}$. CRRS

VERRIUS FLACCUS, Marcus

M. Verrius Flaccus quae extant et Sex. Pompei Festi de verborum significatione lib. $\mathrm{X}[\mathrm{X}]$. In eundem Festum annotationes ... Venetiis, Ex officina S.J. Zilleti, $1560.8^{\circ}$. CRRS

VIRGILIUS MARO, Publius

Georgica P. Virgilii cum novo commentario H. Torrentini. Davetrie, Per me Richardum pafraet, $1496.4^{\circ}$. EJPL 
Virgilius collatione scriptorum Graecorum illustratus ... Antverpiae, Ex officina Plantiniana, 1567. $\dagger$

Opera P. Virgilii Maronis. Pauli Manutii annotationes brevissimae in margine adscriptae. Homeri loca magis insignia quae Virgilius imitatus est. Georgii Fabricii ... observationes Virgilianae lectionis. Antverpiae, Ex officina C. Plantini, 1572. $8^{\circ}$. CRRS

Appendix cum supplemento multorum antehac nunquam excusorum poëmatum; Josephi Scaligeri ... in eandem Appendicem castigationes et commentarii multis in locis auctis ... Lugduni Batavorum, Ex officina Plantiniana, Apud F. Raphelengium, $1595.8^{\circ}$. CRRS

L. Hortensii Montfortii enarrationes ... in XII libros ... Aeneidos. His accessit N. Nascimbaenii ... in sex primos Aeneidos libros ... explanatio ... Nunc demum multis mendis quibus hoc totum opus scatebat ademptis ... Index ... una cum allegoriis Platonicis C. Landini ... in calce ... est adiectus. Basileae, Per S. Henricpetri, 1596. fol. CRRS

Opera, quae quidem exstant omnia, cum ... doctis in Bucolica, Georgica et Aeneida commentariis Tib. Donati et Servii Honorati ... a Georgio Fabricio ... primo collectis et emendatis ... Basileae, Per S. Henricpetri, 1613. fol. CRRS

Elegantes variorum Virgilio-Ovidio-centones de opificio mundi, Christo Deo, Deique matre, SS. Francisco et Car. Borromaeo. Monaci, A. Raphaele Sadlero, 1617. $8^{\circ}$. EJPL

Dell'Eneide del Commendator Annibal Caro. IV impressione. Padova, Appresso Domenico Pasquardi, 1630. $\dagger$

Art Gallery of Ontario 